\title{
Software Implementation of Statistical Modeling \\ of Loads and Accounting \\ for Multimodality of Electric Networks
}

\author{
Alexey A. Gerasimenko and Evgeny V. Puzyrev* \\ Siberian Federal University \\ Krasnoyarsk, Russian Federation
}

Received 22.10.2019, received in revised form 14.12.2019, accepted 21.01.2020

Abstract. Based on earlier authors' software development, a single multifunctional software complex "POTERI V1.1: SETI, REG10PVT" (hereinafter POTERI) was created, which provides accurate and reliable calculation of energy losses in electric networks with a voltage of 6-110 (220) $\mathrm{kV}$. The paper presents the general structure of the POTERI software complex together with a brief description of the software modules SETI, REG10PVT, VES, RES and their performance features. The software modules and algorithms, as well as the whole complex are tested at the network enterprises of the Russian Federation.

Keywords: electric energy losses, software modules, software complex, deterministic, stochastic and combined methods.

Citation: Gerasimenko A.A., Puzyrev E.V. Software implementation of statistical modeling of loads and accounting for multimodality of electric networks, J. Sib. Fed. Univ. Eng. \& Technol., 2020, 13(2), 194-205. DOI: 10.17516/1999-494X-0216

\section{Программная реализация}

статистического моделирования нагрузок

\section{и учета многорежимности электрических сетей}

\author{
А.А. Герасименко, Е.В. Пузырев \\ Сибирский федеральный университет \\ Российская Федеращия, Красноярск
}

Аннотащия. На базе более ранних программных разработок авторов создан единый многофункциональный программный комплекс «POTERI V1.1: SETI, REG10PVT» (далее

(C) Siberian Federal University. All rights reserved

This work is licensed under a Creative Commons Attribution-NonCommercial 4.0 International License (CC BY-NC 4.0).

* Corresponding author E-mail address: puzyrev_ev@mail.ru 
POTERI), позволяющий выполнять расчеты потерь электроэнергии в электрических сетях напряжением 6-110 (220) кВ с высокой точностью и достоверностью. В работе представлена общая структура программного комплекса POTERI, приведено краткое описание программных модулей и особенностей их функционирования: SETI, REG10PVT, VES, RES. Осуществляется апробирование программных модулей, алгоритмов и испытание комплекса в целом в сетевых предприятиях РФ.

Ключевые слова: потери электрической энергии, программные модули, программный комплекс, методы детерминированный, стохастический и комбинированный.

Цитирование: Герасименко, А.А. Программная реализация статистического моделирования нагрузок и учета многорежимности электрических сетей / А.А. Герасименко, Е.В. Пузырев // Журн. Сиб. федер. ун-та. Техника и технологии, 2020. 13(2). С. 194-205. DOI: 10.17516/1999-494X-0216

Правильный учет и анализ, в также снижение потерь электрической энергии (ЭЭ) всегда были и остаются одними из приоритетных задач для сетевых организаций всех уровней и реальной эксплуатационной технологией энергосбережения [1]. Многие электросетевые предприятия рассматривают учет потерь ЭЭ как один из целевых показателей в области повышения их энергоэффективности. Наряду с этим особое место при планировании и анализе режимов работы электрических сетей (ЭС), определении допустимых и фактических балансов ЭЭ, оценке нормированной (нормативной) величины потерь ЭЭ, обосновании и установлении региональных тарифов на ЭЭ занимает развитие и усовершенствование различных способов повышения надежности, эффективности расчета (с высокой точностью и заданной достоверностью) основных параметров всей совокупности возможных режимов работы (многорежимности), определяемой изменением нагрузок ЭС.

Наибольшее распространение получили детерминированные методы расчета потерь ЭЭ [2-4], в меньшей мере - стохастические (вероятностно-статистические) и комбинированные подходы к расчету потерь [5-7].

В России наибольшее применение в области расчета и анализа потерь ЭЭ имеют две многоуровневые программные разработки, реализующие детерминированный подход, - программные комплексы РТП и РАП-стандарт $[8,9]$.

Улучшение эффективности расчетных подходов может быть достигнуто в результате оптимального весового анализа и оценки свойств и возможностей вероятностно-статистических и детерминированных методов расчета потерь ЭЭ и тем самым в определенной мере компенсировать недостатки обоих методов [6].

Программный комплекс POTERI создавали с целью повышения надежности (точности и достоверности) результата расчетов, влияющих на оценку энергоэффективности электросетевых предприятий. Главная особенность комплекса состоит в том, что наряду с детерминированным и стохастическим методами в нем реализован комбинированный подход, уравновешивающий достоинства и недостатки каждого из методов. В комплексе обобщен опыт программирования и использования предыдущих программных разработок.

\section{Основные расчетные выражения}

Центральной проблемой при определении потерь ЭЭ является учет всей совокупности режимов (многорежимности), вызванной изменением нагрузок на заданном времен- 
ном интервале (сутки, месяц, квартал) $T$. Для всей схемы сети, имеющей $m$ продольных элементов и $n+1$ узлов, нагрузочные потери ЭЭ определяются непосредственным суммированием потерь мощности $\Delta P$ на всех $d$ интервалах стационарности (усреднения) $\Delta t$ графиков нагрузок расчетного периода $T$, что описывается следующими принципиальными выражениями [3]:

$$
\Delta W_{\Sigma}^{\text {н }}=3 \sum_{j=1}^{m} R_{j} \int_{0}^{T} I_{j}^{2}(t) d t=\sum_{j=1}^{m} \int_{0}^{T} \Delta P_{j}(t) d t=\sum_{i, j}^{n+1} \int_{0}^{T} \Delta P_{i j}(t) d t \approx \sum_{i, j}^{n+1} \sum_{s}^{d} \Delta P_{i j s} \Delta t_{s},
$$

где $d=T / \Delta t$ - число интервалов постоянства (стационарности) графиков электрических нагрузок; $\Delta P_{i j}$ - потери мощности на участке $i-j$ электрической сети для данного интервала постоянства $s$.

Реализация (1), выполняемая на основе серии расчетов установившихся режимов и дающая наиболее точные значения потерь ЭЭ, чрезмерно трудоемка, в том числе как из-за большого объема, так и случайного и частично неопределенного характера части исходной информации, особенно для сетей 6-35 кВ. В программном комплексе POTERI, объединяющем под общее управление ряд программно-расчетных модулей, алгоритмы базируются в основном на одном расчете установившегося режима для средних нагрузок. При этом реализованы три направления учета всей совокупности режимов и, соответственно, расчета потерь ЭЭ.

1. Детерминированный алгоритм, известный как метод средних нагрузок, использует данные системы головного учета - отпуск ЭЭ в сеть, потребленную ЭЭ, эквивалентное напряжение источника - и реализуется в следующем виде:

$$
\Delta W_{\partial e m}=3 k_{\phi}^{2} \sum_{j=1}^{m} I_{c p j}^{2} R_{j} T=\left[\sum_{j=1}^{m} \Delta P_{c p j}+\left(k_{\phi}^{2}-1\right) \Delta P_{c p}\right] T=M \Delta W+\sigma \Delta W,
$$

где $I_{c p j}$ - среднее значение тока $M \Delta W, \sigma \Delta W$ - основная и дисперсионная составляющие потерь ЭЭ $[3,4]$.

Коэффициент формы $k_{\phi}$, учитывающий информацию о множестве режимов, определяется по данным отпуска ЭЭ $W_{P}, W_{Q}$ в сеть $k_{\phi}=\frac{\sqrt{d}}{\sqrt{W_{P}^{2}+W_{Q}^{2}}}\left(\sqrt{\sum_{j=1}^{d} W_{P j}^{2}+\sum_{j=1}^{d} W_{Q j}^{2}}\right)$ или вычисляется итерационно через дисперсионную составляющую потерь $\sigma \Delta W$, определяемую вероятностностатистическим методом при комбинированом подходе $k_{\phi}^{2}=1+\frac{\sigma \Delta W}{M \Delta W}$.

2. Вероятностно-статистический метод, базирующийся на факторной модели электрических нагрузок и модифицированной стохастической модели анализа многорежимности, представлен выражением:

$$
\begin{gathered}
\Delta W_{\text {s.c. }} \approx\left[\Delta P(M U, M \delta)+\left(\frac{1}{2} \sum_{i=1}^{n} \sum_{j=1}^{n} k\left(U_{i} U_{j}\right) \frac{\partial^{2} \Delta P}{\partial U_{i} \partial U_{j}}+\sum_{i=1}^{n} \sum_{j=1}^{n} k\left(U_{i} \delta_{j}\right) \frac{\partial^{2} \Delta P}{\partial U_{i} \partial \delta_{j}}+\right.\right. \\
\left.\left.+\frac{1}{2} \sum_{i=1}^{n} \sum_{j=1}^{n} k\left(\delta_{i} \delta_{j}\right) \frac{\partial^{2} \Delta P}{\partial \delta_{i} \partial \delta_{j}}\right)\right] \cdot T=[\Delta P(M U, M \delta)+\sigma(\Delta P)] \cdot T=M \Delta W+\sigma \Delta W \\
-196-
\end{gathered}
$$


где $\Delta P(M U, M \delta), k\left(\delta_{i} \delta_{j}\right), k\left(\delta_{i} U_{j}\right), k\left(U_{i} U_{j}\right)$ - основная составляющая потерь мощности, корреляционные моменты, вычисленные через модули и фазы напряжения $M U, M \delta$ в точке, соответствующей математическим ожиданиям электрических нагрузок; $\frac{\partial^{2} \Delta P}{\partial U_{i} \partial U_{j}}, \frac{\partial^{2} \Delta P}{\partial U_{i} \partial \delta_{j}}, \frac{\partial^{2} \Delta P}{\partial \delta_{i} \partial \delta_{j}}$ вторые производные выражения потерь мощности по соответствующим параметрам, вычисленные относительно той же точки; $\sigma(\Delta P)$ - дисперсионная составляющая потерь активной мощности $[5,10]$.

Корреляционные моменты $k\left(V_{i} V_{j}\right), k\left(V_{i} \delta_{j}\right), k\left(\delta_{i} \delta_{j}\right)$ модулей и фаз напряжений, вычисленные относительно параметров $\mathrm{M} \bar{V}, M \bar{\delta}$, находятся через вспомогательные коэффициенты, получаемые из $K=2-3$ решений систем линейных уравнений (СЛУ), аналогичных системам линеаризованных уравнений узловых напряжений

$$
[J]\left[\begin{array}{l}
\gamma_{k}^{\prime} \\
\gamma_{k}^{\prime \prime}
\end{array}\right]=\left[\begin{array}{l}
\overline{\vartheta_{k}^{\prime}} \\
\overline{\vartheta_{k}^{\prime \prime}}
\end{array}\right], k=1, \bar{K} .
$$

Компоненты векторов $\vartheta_{k}^{\prime}, \vartheta_{k}^{\prime \prime}$ правых частей СЛУ (4), учитывающие отклонения активных и реактивных мощностей узлов от соответствующих математических ожиданий, находят через центрированные величины $\Delta P_{i j}, \Delta Q_{i j}$ нагрузок соответствующих узлов и составляющие $G_{k j}$ факторной модели нагрузок $\vartheta_{k i}^{\prime}=\frac{1}{d} \sum_{j=1}^{d} G_{k j} \cdot \Delta P_{i j}, \vartheta_{k i}^{\prime \prime}=\frac{1}{d} \sum_{j=1}^{d} G_{k j} \cdot \Delta Q_{i j}, i=\overline{1, n}$.

3. Комбинированный подход основан на непосредственном использовании результатов детерминированного $\Delta W_{\text {дет }}$ и стохастического $\Delta W_{\text {в.c. }}$ алгоритмов расчета потерь ЭЭ в составе средневзвешенной величины $[6,11]$ :

$$
\Delta W_{\text {pacu }}=\Delta W_{\text {деm }} \cdot \eta+\Delta W_{\text {в.c. }} \cdot \alpha
$$

где $\alpha, \eta$ - оптимальные по критерию минимума ошибки значения весовых коэффициентов, связанных соотношением $\eta=1-\alpha$, определяющих вес (участие) результата соответственно стохастическому и детерминированному алгоритмам в расчетной средневзвешенной величине потерь ЭЭ:

$$
\Delta W_{p a c u}=\Delta W_{\text {деm }} \cdot(1-\alpha)+\Delta W_{\text {в.c. }} \cdot \alpha .
$$

Оптимальное сочетание результатов уточненного детерминированного и стохастического методов обосновано экспериментально [11].

В программном комплексе POTERI выполнен учет ряда схемно-структурных и режимноатмосферных факторов: внутри интервального изменения электропотребления и температуры воздуха, структуры схемы ЭС, скорости ветровой нагрузки, температуры проводов и жил кабелей $[4,12]$.

\section{Краткое описание программ REG10PVT и SETI}

Для создания программного комплекса POTERI в первую очередь по исходным текстам была выполнена сборка и отладка отдельных программ REG10PVT и SETI, написанных на язы-

$$
-197-
$$


ках программирования FORTRAN и PASCAL, которые длительное время использовались для научных и инженерных вычислений. На рис. 1 и 2 представлены главные окна и окна ввода исходных данных одних из первых версий программ REG10PVT и SETI $[3,4]$.

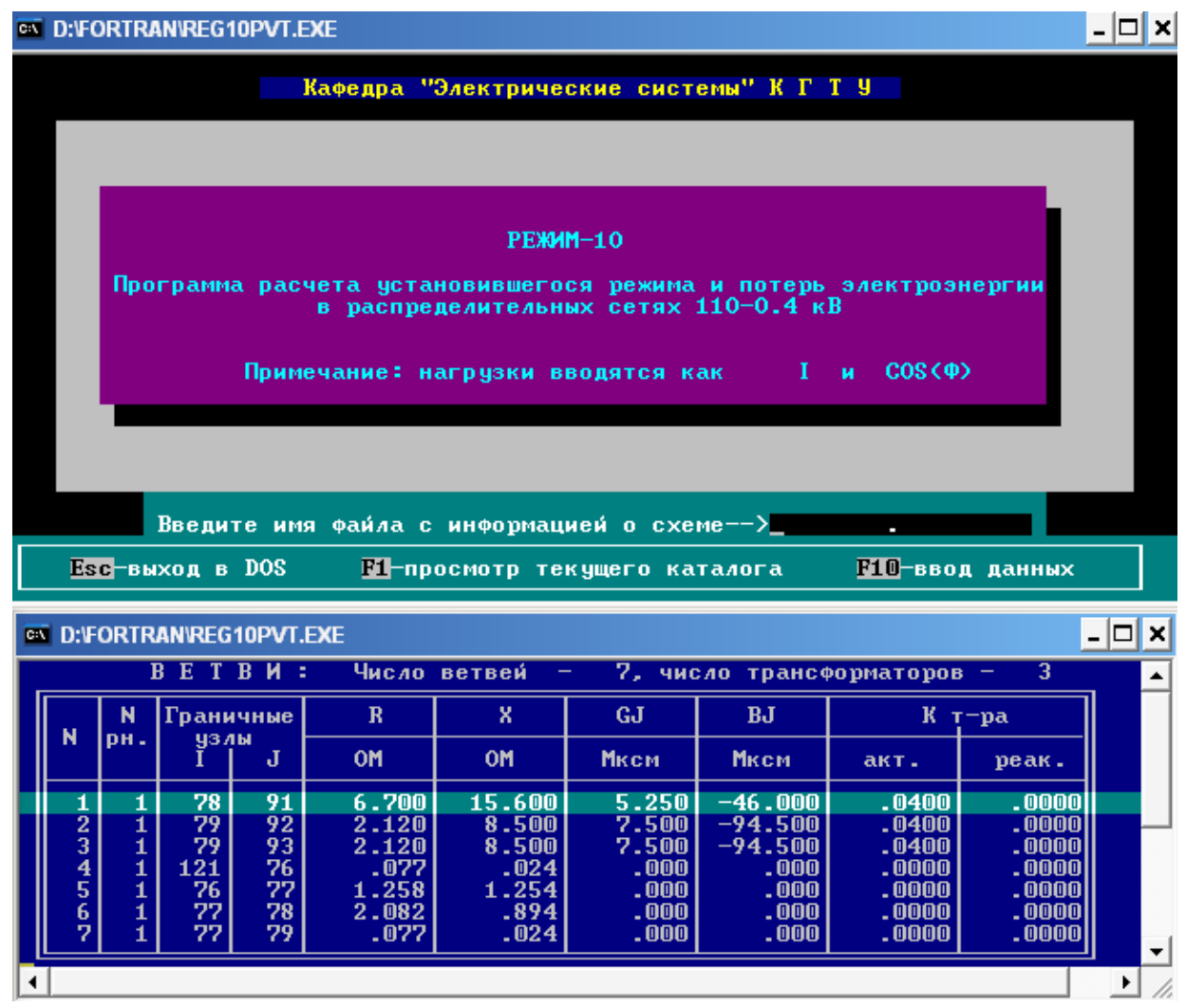

Рис. 1. Главное окно и окно ввода исходных данных программы REG10PVT

Fig. 1. Main window and input window of REG10PVT program

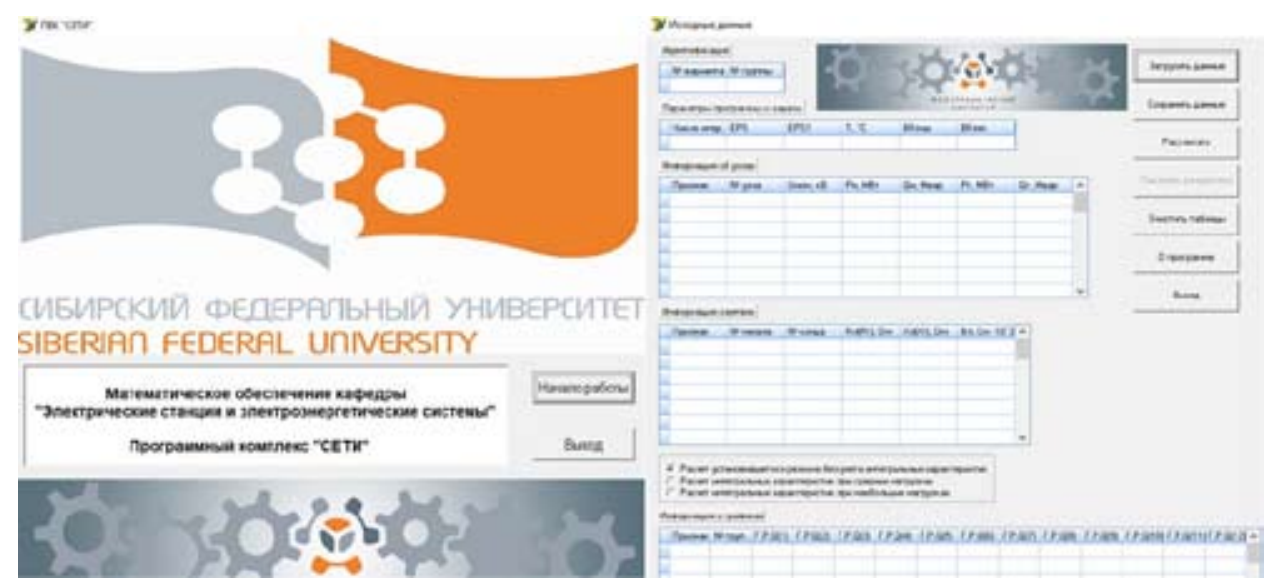

Рис. 2. Главное окно и окно ввода исходных данных программы SETI

Fig. 2. The main window and the input window of the source program data SETI

$$
-198-
$$


Программа REG10PVT состоит из следующих частей:

- программа расчета установившихся режимов и потерь ЭЭ в отдельном участке сети (фидере);

- программа (утилита) обновления информации по всем фидерам предприятия (пропуск ЭЭ, количество часов работы, температура воздуха и др.);

- программа расчета потерь ЭЭ по всей базе данных фидеров ЭС и формирование результата расчета в форме, приближенной к отчетной.

Расчет установившихся режимов и интегральных характеристик всей совокупности режимов электрической сети на произвольном временном интервале реализованы в программе SETI [5]. Основу соответствующих алгоритмов составляет метод Ньютона и вероятностностатистическая факторная модель электрических нагрузок - обобщенные графики нагрузок (ОГН) [10].

Большинство библиотек FORTRAN являются доступными в исходных кодах, они хорошо документированы, отлажены и весьма эффективны в практическом использовании. Поэтому изменять, а тем более переписывать их на другие современные языки программирования - задача достаточно трудоемкая и сопровождается, как правило, скрытыми ошибками. На фоне быстрого обновления технологий, средств редактирования, операционных систем разработчики не всегда учитывают совместимость новых технологий с программными продуктами, созданными ранее при имеющемся уровне развития техники и технологий. В связи с этим становится актуальной проблема адаптации ранее апробированных и успешно прошедших расчетные испытания программных продуктов к современным средствам программного обеспечения. Тем не менее авторами была произведена успешная попытка конвертирования кодов FORTRAN на более современный язык программирования DILPHI и создания нового удобного интерфейса.

\section{Общая характеристика}

\section{и структура программного комплекса POTERI}

Поэтапное изучение работы отдельных программ и проработка алгоритмов, их доработка и усовершенствование способствовали созданию нового единого программного комплекса POTERI, который имеет несколько версий [12]. Каждая из версий комплекса отличается различным набором функциональных возможностей программных модулей. Последняя версия комплекса построена на базе четырех программных модулей SETI, REG10PVT, VES, RES (рис. 3).

B совокупности комплекс POTERI имеет широкий функционал, поскольку реализует различные методы по расчету и учету потерь ЭЭ - детерминированный, стохастический (вероятностно-статистический) и комбинированный.

Далее кратко представлены возможности сформированного программного комплекса POTERI. Подробное описание по работе пользователя с комплексом и его функционированию изложено в инструктивных указаниях к каждому из программных модулей.

На рис. 4 приведено главное окно программного комплекса POTERI версии 2.3 и основные окна программных модулей SETI, REG10PVT, VES.

В зависимости от имеющейся исходной информации и цели расчета у пользователя есть возможность выполнить расчет любым из трех способов. Для этого необходимо заранее подго- 


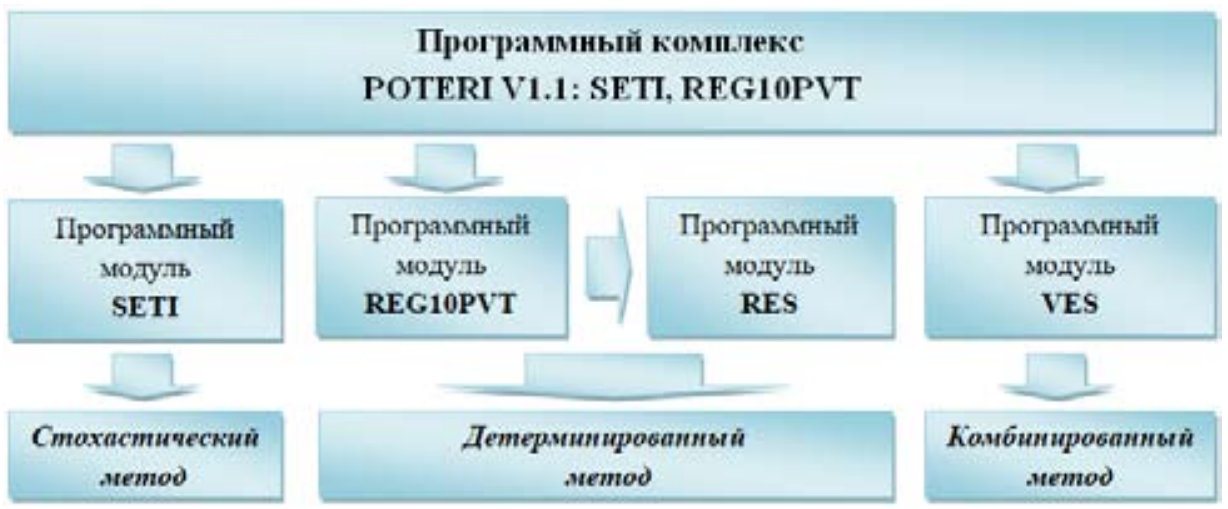

Рис. 3. Укрупненная модульная структура программного комплекса POTERI

Fig. 3. Integrated modular structure of the software complex POTERI

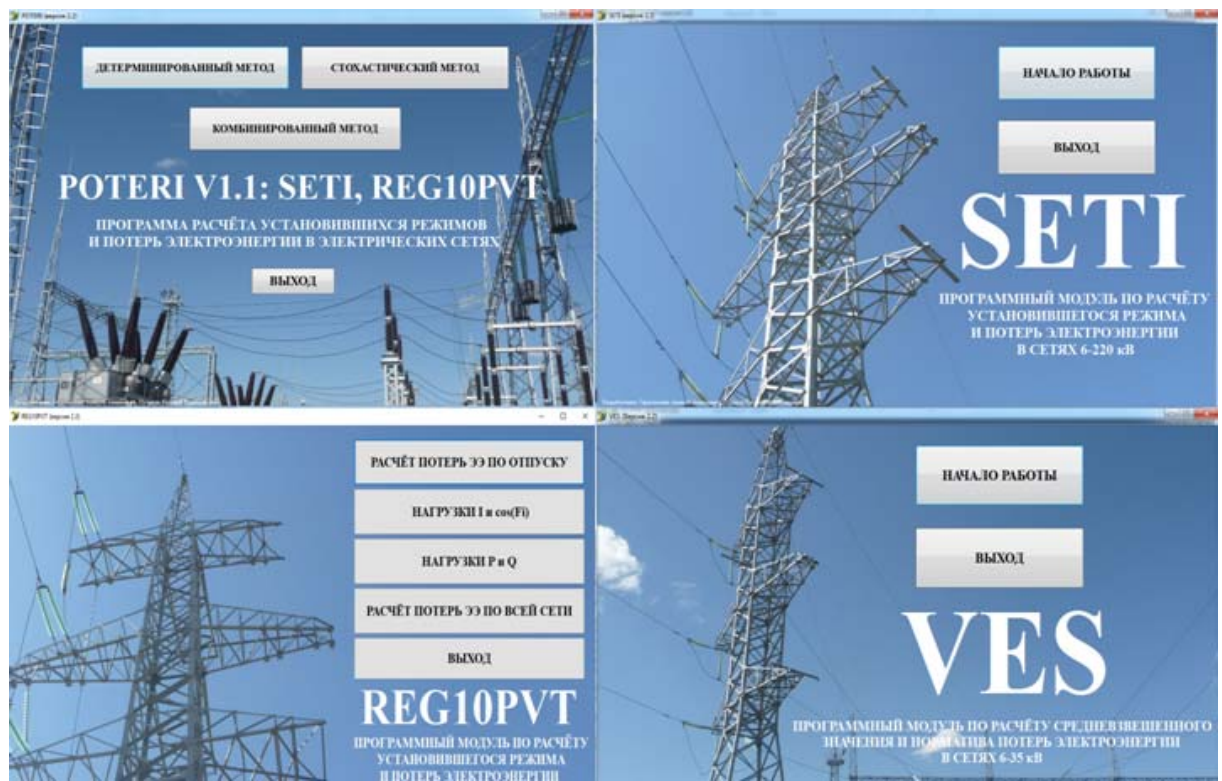

Рис. 4. Главное окно и основные окна программного комплекса POTERI

Fig. 4. The main window and the main windows of the software complex POTERI

товить исходный файл, который для каждого из модулей имеет свои особенности построения и ввода параметров схемы замещения.

Расчет потерь электроэнергии детерминированным способом (вход «Детерминированный метод») организуется с помощью основного диалогового окна программного модуля REG10PVT. В программном модуле REG10PVT реализована возможность расчета параметров установившегося режима сети по нагрузкам, заданным значениями активной и реактивной мощностей (Р и Q) - вход (кнопка) «НАГРУЗКИ Р и $\mathrm{Q} »$ или тока и коэффициента мощности (I и $\cos \varphi)$ - вход (кнопка) «НАГРУЗКИ I и $\cos (\mathrm{Fi}) »$. В программном модуле возможно выполнение расчета потерь электроэнергии как для отдельного участка сети (вход «РАСЧЕТ ПОТЕРЬ 
ЭЭ ПО ОТПУСКУ»), так и в целом по всей сети напряжением 6-35 кВ для неограниченного количества фрагментов, составляющих в совокупности рассматриваемую сеть (вход «РАСЧЕТ ПОТЕРЬ ЭЭ ПО ВСЕЙ СЕТИ») - Программный модуль RES, структурно интегрированный в программный модуль REG10PVT [13].

В программном модуле SETI практически реализована статистическая методология сжатого нетрудоемкого моделирования и учета многорежимности ЭС: в основе факторное моделирование матрицы корреляционных моментов (МКМ) методом главных компонент с получением ОГН, ограниченный набор которых позволяет нетрудоемко, приемлемо точно и надежно решать задачи расчета и нормирования потерь ЭЭ, а также определять другие интегральные характеристики (диапазоны и диаграммы (графики) изменения напряжений в узлах сети и реактивной мощности источников), поддерживающие решения ряда технических задач анализа режимов и управления ими $[5,10]$. В данном модуле возможно выполнение трех видов расчетов, выбор которых определяется целью расчета и имеющийся исходной информацией: расчет установившихся режимов - узловые нагрузки в мощностях P, Q; расчет потерь ЭЭ и интегральных характеристик при задании нагрузок средними значениями $\mathrm{P}, \mathrm{Q} ;$ расчет потерь ЭЭ и интегральных характеристик при задании нагрузок максимальными значениями $\mathrm{P}, \mathrm{Q}$.

Следует отметить, что расчет интегральных характеристик возможен при вводе суточных графиков активных и реактивных мощностей $\mathrm{P}(\mathrm{t}), \mathrm{Q}(\mathrm{t})$ в узлах в явном виде или после считывания отраслевых (типовых) графиков из базы данных и обобщенных факторных моделей - ортогональных графиков нагрузки Г(t) [5]. Особенностью программного модуля SETI является то, что для расчета потерь ЭЭ стохастическим методом помимо трех ОГН, встроенных в модуль, дополнительно реализована возможность ввода отдельных ОГН, вновь полученных после статистической обработки отраслевых графиков различных распределительных систем, что существенно расширяет возможности программного модуля в направлении повышения точности и надежности результатов расчета потерь ЭЭ.

На рис. 5 приведена обобщенная схема работы программного модуля SETI, функционально состоящего из отдельных программных блоков-алгоритмов:

APOID - контроля ошибок данных; WZDU - ввод исходных данных; FORMY - формирования связных списков матрицы узловых проводимостей; RANG - определения рангов узлов, перенумерации узлов, ветвей; TREANG - оптимального упорядоченного исключения узлов; NUTON - решения системы уравнений узловых напряжений (УУН); OPRNEW - вычисления невязок системы УУН; LZB - решения системы линейных уравнений с нижней треугольной матрицей; WUZ - решения системы линейных уравнений с верхней треугольной матрицей; TBL - вывода результатов расчета установившегося режима сети по математическим ожиданиям нагрузок; GAMMA - вычисления собственных векторов и расчетных переменных $\gamma$; POTERI - определения дисперсионной составляющей потерь ЭЭ; DIAP - определения суточного режима напряжения в узлах сети; STAR - проверки соответствия параметров схемы; BNGR - работы с суточными и обобщенными графиками нагрузки.

Программная реализация комбинированного метода выполнена на основе результатов эксперимента по поиску оптимального сочетания уточненного детерминированного и стохастического методов. Для вычисления средневзвешенного значения необходимо произвести расчеты потерь ЭЭ с помощью программных модулей REG10PVT и SETI, сохранив в отдельной

$$
-201-
$$




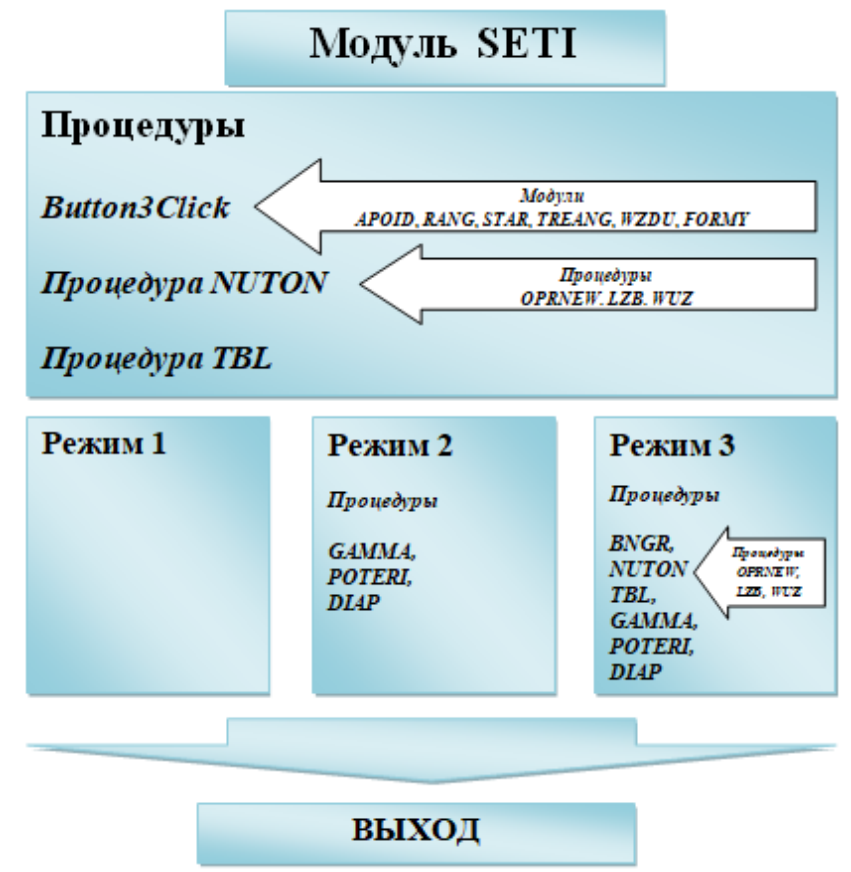

Рис. 5. Обобщенная схема функционирования программного модуля SETI

Fig. 5. Generalized scheme of SETI software module functioning

папке протоколы расчета в формате *.csv. C помощью программного модуля VES необходимо выбрать соответствующие протоколы и одно из возможных весовых сочетаний оптимального диапазона $\alpha=[0,7 ; 0,8]$ результатов детерминированного и стохастического алгоритмов. В модуле VES программно реализован усовершенствованный алгоритм расчета и оценки нормативных значений потерь ЭЭ на базе комбинированного подхода $[6,11,14,15]$.

В итоге выполнен анализ структуры и модернизация ранее созданных на языке FORTRAN программ SETI и REG10PVT и составляющих их программных блоков с переходом на алгоритмический язык DELPHI с тщательной проработкой и улучшением программных возможностей расчета интегральных характеристик через восстанавливаемые средние нагрузки. Изменен принцип составления файлов исходных данных и их считывание. Значительно переработан и сформирован новый пользовательский интерфейс, отвечающий за удобный ввод, вывод и сохранение данных и сопровождаемый подробной пользовательской инструкцией.

Программный комплекс POTERI предназначен для расчета технических потерь ЭЭ в ЭС с номинальным напряжением 6-110 кВ, а также анализа установившихся электрических режимов сетей напряжением 0,38-110 кВ в диалоговом режиме и выводе результатов расчетов в табличной форме. Комплекс позволяет рассчитывать потери ЭЭ и их структуру на различных уровнях обобщения ЭС: отдельно распределительная линия (фидер), подстанция, район электрической сети и предприятия в целом. Результаты обобщаются в виде отпущенной в сеть ЭЭ, степени загрузки (использование) ЭС, параметров базового электрического режима. В структуре потерь ЭЭ выделены составляющие: потери в линиях, трансформаторах, в том числе нагрузочные, и потери холостого режима. При анализе потерь наряду с детерминиро- 


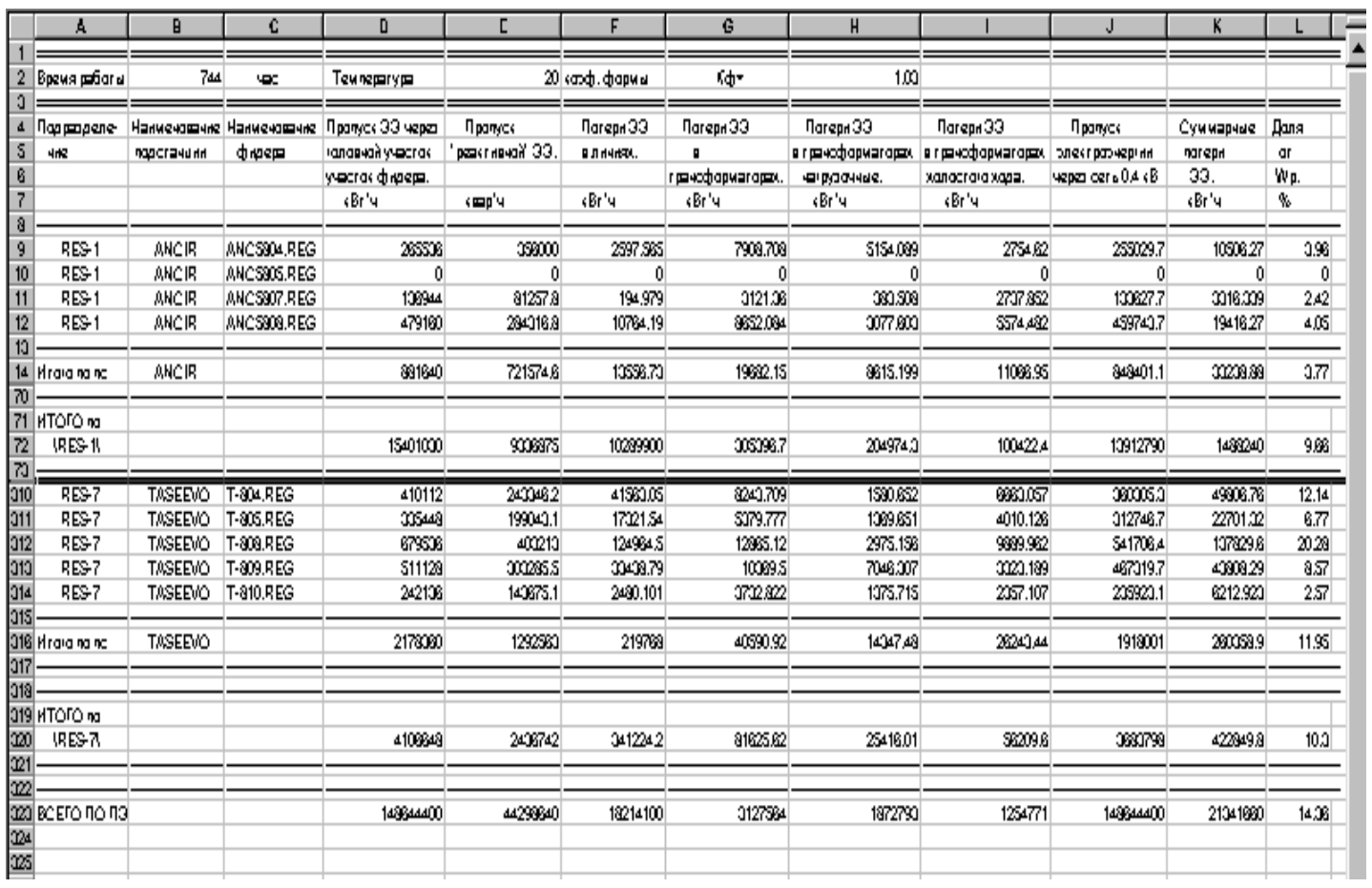

Рис. 6. Пример вывода результатов расчета потерь электроэнергии по предприятию

Fig. 6. Example of output results of calculation of losses of EE on the enterprise

ванной величиной потерь ЭЭ определяется интервал, в котором они находятся с определенной достоверностью. На основании исходной информации и результатов расчетов формируются файлы отчета требуемой структуры в текстовом формате, содержащие таблицы, совместимые с форматом EXCEL (рис. 6). Таблицы компактны и удобны для восприятия, предусмотрена возможность их просмотра на экране, записи на носители информации и вывода на печатающие устройства.

Примеры расчетов с помощью представленных отдельных программ REG10PVT и SETI и программного комплекса POTERI приведены в $[3-5,10,11,16]$.

Программный комплекс POTERI имеет государственную регистрацию в реестре программ для ЭВМ [17], прошел апробацию в сетевых организациях, используется для решения ряда задач эксплуатации и функционирования электрических сетей, готов к регулярному применению в учебных и научных целях.

\section{Основные выводы и результаты}

1. Сняты ограничения в части совместимости программ REG10PVT и SETI, написанных на языке программирования FORTRAN, с различными операционными системами, используемыми на предприятиях и в организациях. Программный комплекс POTERI совместим с различными операционными системами: XP / VISTA / WINDOWS 7 / WINDOWS 8 / WINDOWS 10.

2. Разработан единый многофункциональный программный комплекс «POTERIV1.1: SETI, REG10PVT» и в целом создана математическая, алгоритмическая и программная основа

$$
-203-
$$


для разработки программно-вычислительного аппарата расчета, анализа и нормирования потерь ЭЭ высокой надежности общесистемного (отраслевого) уровня.

\section{Список литературы / References}

[1] Воротницкий В.Э., Кутовой Г.П., Овсейчук В.А. Снижение потерь электроэнергии. Стратегический путь повышения энергетической эффективности сетей. Новости электротехники. 2015, 4(94). 22 c. [Vorotnitsky V.E., Kutovoy G.P., Ovseichuk V.A. Reduction of power losses. Strategic way to improve the energy efficiency of networks. News of electrical engineering. 2015, 4(94). 22 p. (in Russian)]

[2] Железко Ю.С., Артемьев А.В., Савченко О.В. Расчет, анализ, нормирование потерь электроэнергии в электрических сетях. М.: Изд-во НЦЭНАС, 2004, 290 c. [Zelezko U.S., Artemyev A.V., Savchenko O.V. Calculation, analysis, regulation of electricity losses in electric networks. Moscow, Publishing house of the NTS ENAS, 2004, 290 p. (in Russian)]

[3] Герасименко А.А., Тимофеев Г.С. Методика, алгоритм и программа расчета технических потерь электроэнергии в распределительных сетях энергосистем. Вестник электроэнергетики. М.: ИНФОРМЭНЕРГО, 2001, (4), 74 с. [Gerasimenko A.A., Timofeev G.S. Methods, algorithm and software for calculation of technical losses of electricity in distribution networks power systems. The Journal of electricity, Moscow, INFORMENERGO, 2001, (4), 74 p. (in Russian)]

[4] Герасименко А.А., Куценов Д.А., Тимофеев Г.С. Уточнение технической и коммерческой составляющих потерь электроэнергии в распределительных сетях. Известия вузов. Электромеханика, 2005, (5), 38-43 [Gerasimenko A.A., Kucenov D.A., Timofeev G.S. Clarification of technical and commercial components of energy losses in distribution networks. News of Higher educational institutions. Electrician, 2005, (5), 38-43 (in Russian)]

[5] Герасименко А.А., Шульгин И.В. Стохастический метод расчета нагрузочных потерь электроэнергии в распределительных электрических сетях. Электрические станции, 2013, (4), 44-59 [Gerasimenko A.A., Shulgin I.V. Stochastic method of calculating the load losses of electricity in distribution networks. Power stations, 2013, (4), 44-59 (in Russian)]

[6] Gerasimenko A.A., Puzyrev E.V. The Combined Presentation of Deterministic and Stochastic Approaches in the Algorithm of Calculation of Energy Losses in Electric Networks, J. Sib. Fed. Univ. Eng. technol., 2017, 10(1), 6-16.

[7] Xin Kaiyuan, Yang Yuhua, Chen Fu. An advanced algorithm based on combination of GA with BP to energy loss of distribution system [J]. Proceedings of the CSEE, 2002, 22(2), 79-82.

[8] Программный комплекс «РАП-стандарт» [Электронный ресурс] - Режим доступа: http://www.rap-standart.ru - Заглавие с экрана. [Program complex «RAP-standard» [Electronic resource] - Access: http://www.rap-standart.ru]

[9] Программный комплекс «РТП» [Электронный ресурc]. - Режим доступа: http://www. rtp3.ru/about.htm. [Program complex «RTP» [Electronic resource] - Access: http://www.rtp3.ru/ about.htm.]

[10] Герасименко А.А., Нешатаев В.Б., Шульгин И.В. Расчет потерь электроэнергии в распределительных электрических сетях на основе вероятностно-статистического моделирования электрических нагрузок. Изв. вузов. Электромеханика, 2011, (1), 71-77 [Gerasimenko A.А., Neshataev V.B., Shulgin I.V. Calculation of energy losses in electrical distribution networks based 
on probabilistic-statistical modeling of electrical loads. News of Higher educational institutions. Electrician, 2011, (1), 71-77 (in Russian)]

[11] Герасименко А.А., Пузырев Е.В. Комбинированное объединение детерминированного и стохастического методов в алгоритме расчета потерь электроэнергии. Научно-технический журнал «ЭЛЕКТРО. Электротехника, электроэнергетика, электротехническая промышленность», 2017, 3, 12-16. [Gerasimenko A.A., Puzyrev E.V. Combined combination of deterministic and stochastic methods in the algorithm of calculation of electric power losses. Scientific and technical journal «ELECTRO. Electrical engineering, electric power industry, electrotechnical industry», 2017, 3, 12-16. (in Russian)]

[12] Герасименко А.А., Пузырев Е.В. Software implementation of deterministic and stochastic calculation methods of electric energy losses in electrical distribution networks, Znanstvena misel journal, 2018, 1(14), 49-57 [Gerasimenko A.A., Puzyrev E.V. Software implementation of deterministic and stochastic calculation methods of electric energy losses in electrical distribution networks, Znanstvena misel journal, 2018, 1(14), 49-57 (in Russian)]

[13] Герасименко А.А., Пузырев Е.В. Программный модуль «RES» программного комплекса «POTERI» расчета потерь электроэнергии в распределительных сетях. Технические науки: проблемы и решения. Сб. ст. по материалам ХІХ Международной научно-практической конференции. М.: Интернаука, 2019, 1(17), 108-114 [Gerasimenko A.A., Puzyrev E.V. Software module «RES» of the software complex «POTERI» for calculating power losses in distribution networks. Technical Sciences: problems and solutions: collection of articles on the materials of the XIX International scientific and practical conference. M., Internauka, 2019, 1(17), 108-114 (in Russian)]

[14] Герасименко А.А., Пузырев Е.В. Программный модуль «VES» комбинированного расчета потерь электрической энергии программы «POTERI V1.1: SETI, REG10PVT». Проблемы и перспективы развития электроэнергетики и электротехники. Сб. ст. Всероссийской научно-практической конферениии. Казань, 2019, 3-8 [Gerasimenko A.A., Puzyrev E.V. Program module «VES» of combined calculation of electric energy losses of the program «POTERI V1. 1: SETI, REG10PVT». Problems and prospects of development of electric power industry and electrical engineering. Collection of articles of the all-Russian scientific and practical conference, Kazan, 2019, 3-8 (in Russian)]

[15] Gerasimenko A.A., Puzyrev E.V. The algorithm of calculation of standard value of losses of the electric power in distribution networks is Improved. International scientific conference «scientific research of SCO countries: synergy and integration», Beijing, 2019, 161-167.

[16] Тимофеев Г.С. Комплексный учет схемно-структурных и режсимо-атмосферных факторов при расчете потерь электроэнергии в распределительных сетях энергосистем, автореф. дисс. ...канд. техн. наук. Красноярск, 2011, 21 с. [Timofeev G.S. Complex accounting of circuitstructural and regime-atmospheric factors at calculation of losses of the electric power in distribution networks of power systems, Thesis ... cand. of tech. Sci. Krasnoyarsk, 2011, 21 p. (in Russian)]

[17] Герасименко А.А., Пузырев Е.В. Программа расчета установившихся режимов и потерь электрической энергии в электрических сетях «POTERI V1.1: SETI, REG10PVT». Российская Федерация. № 2017661621 от 17 октября 2017 г. [Gerasimenko A.A., Puzyrev E.V. Program of calculation of steady-state modes and losses of electric energy in electric networks «POTERI V1. 1: SETI, REG10PVT». Russian Federation. No. 2017661621 of October 17, 2017 (in Russian)]

$$
-205-
$$

